\title{
Plasma-Assisted Fabrication of Self-Assembled Phospholipid Layer onto Polymer Surface and Its Characterization
}

\author{
Shin-ichi Kondo ${ }^{1 *}$, Yasushi Sasai ${ }^{1}$, Yukinori Yamauchi ${ }^{2}$, Masayuki Kuzuya ${ }^{2}$ \\ ${ }^{I}$ Laboratory of Pharmaceutical Physical Chemistry, Gifu Pharmaceutical \\ University, 5-6-1 Mitahora-Higashi, Gifu 502-8585, Japan \\ ${ }^{2}$ Department of Pharmaceutical Physical Chemistry, Faculty of \\ Pharmaceutical Sciences, Matsuyama University, 4-2 Bunkyo-cho, Matsuyama, \\ Ehime 790-8578, Japan
}

\section{Keywords: self-assembled phospholipid layer, plasma irradiation, low density polyethylene}

\section{Introduction}

Considerable interest has focused on the immobilization of functional molecules, such as DNA, enzyme and protein, onto the water-insoluble supports, which has been used as a biochip or bioreactor.[1-3] Methods used for the immobilization of enzymes and proteins fall into four main categories: 1) physical adsorption onto an inert support, 2) inclusion in the lattices, 3) cross-linking of the protein with a bifunctional reagent, and 4) covalent binding to a reactive insoluble support. [4-6] The covalent binding method has several advantages and disadvantages. Covalent binding is usually thought to be the stable method to prevent the elution of protein from the support. A great deal of flexibility in designing an immobilized protein with specific physical and chemical properties are allowed by selecting carrier materials and binding method. On the other hand, covalent binding methods are the relatively expensive and complicated in procedures involved. Activity yields may be low due to exposure of the proteins to harsh environments or toxic reagent. Active site may also be modified through the chemical reactions used to create covalent bonding.

We have recently reported a novel method to introduce a durable surface wettability and minimize its decay with time on several hydrophobic polymers, such as polyethylene-naphthalate, polyethylene, nylon-12 (Fig.1).[7-11] This method involves sorption of methylvinylether-maleic anhydride copolymer (VEMA) into the surface layer and immobilization by a plasma-assisted cross-link reaction. Hydrolysis of VEMA follows, to generate hydrophilic carboxyl groups on the surface. Durable surface hydrophilicity introduced in this way has been confirmed both by the measurement of the water contact angle and by demonstration of the long-term stability of the surface lubricity on the urethane-made catheter.

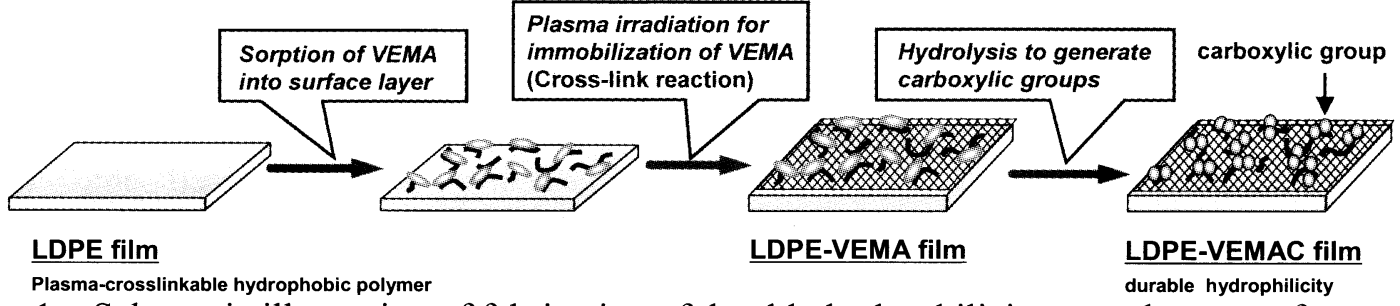

Fig. 1 Schematic illustration of fabrication of durable hydrophilicity on polymer surface 
The cytomembrane is a lipid bilayer that serves to separate the environment inside a cell from that outside. Various types of enzymes and proteins inside the cell demonstrate the highly specific function. Therefore, it is considered that lipid layer would not make proteins denatured. However, it is difficult to obtain the stable phospholipid layer by the immersion of hydrophobic polymeric material into the suspension of phospholipid. Phosopholipids adsorbed on the polymeric material may easily desorb from the surface due to the weak interaction between them. If alkyl groups are introduced as anchoring units (grafted alkyl groups) on the polymeric material surface as shown in Fig. 2, the self-assemble phospholipid layer may be formed by the immersion of polymeric material into the phospholipid suspension due to the hydrophobic interaction between the grafted alkyl groups and phospholipids. We report a novel fabrication of self-assembled phospholipid layer on hydrophobic polymer surface by plasma technique. The procedure is as follows: (Fig. 2) Alkyl amines, such as hexamethylene diamine (HMDA), were immobilized on LDPE-VEMAC film by condensation reaction (LDPE-HE). This film was immersed into the phospholipid suspension to fabricate self-assembled layer of phosphatidyl choline (PC)(LDPE-PC-SA film). The phospholipid layer formed was characterized by ATR-FTIR spectrum and AFM measurement. We also tried to fabricate the self-assembled phospholipid layer containing fatty acid on LDPE-HE film. The fatty acid was introduced as a scaffold to immobilize biomolecules. A model protein, albumin, was immobilized onto this film. The fluidity of this film was also estimated.

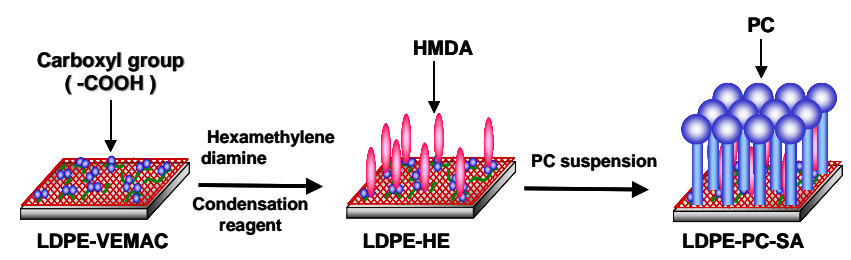

Fig. 2 Schematic illustration for the fabrication of self-assembled phospholipid layer onto a hydrophobic material.

\section{Experimental}

\subsection{Preparation of LDPE-HE film}

An LDPE-VEMAC film was prepared according to the method reported previously. A LDPE film (10 $\mathrm{mm} \times 30 \mathrm{~mm})$ was soaked in cyclohexanone solution containing 2\% VEMA and 5\% p-xylene for $6 \mathrm{~h}$ at $60{ }^{\circ} \mathrm{C}$ and dried in vacuo overnight. The treated LDPE film was subjected to $\mathrm{Ar}$ plasma-irradiation to immobilize VEMA onto the LDPE surface layer. The plasma state was generated by radio frequency discharge of inductive coupling with a five loop antenna at $13.56 \mathrm{MHz}$ and a $20 \mathrm{~W}$ power supply. The flow volume $(50 \mathrm{ml} / \mathrm{min})$ and pressure (0.5 Torr) of argon gas were controlled by changing the evacuating speed. The sample film was placed on a glass-tripod in a reaction chamber to ensure homogeneous exposure to plasma gas.

After plasma-irradiation, the hydrolysis of maleic anhydride linkage in VEMA was conducted to obtain LDPE-VEMAC film. The LDPE-VEMAC film was soaked in a mixture of water $(5 \mathrm{ml})$ and $0.25 \mathrm{~mol} / \mathrm{l} \mathrm{N}$-(3-dimethylaminopropyl-N'-ethylcarbodiimide hydrochloride (EDC) solution (1ml) at $30{ }^{\circ} \mathrm{C}$ for $2 \mathrm{~h}$. After adding $0.25 \mathrm{~mol} / \mathrm{l}$ HMDA solution (1ml), the film was kept at $30{ }^{\circ} \mathrm{C}$ for $20 \mathrm{~h}$. After then, the film was washed with water and dried in vacuo.

2.2 Fabrication of self-assembled phospholipid layer

The LDPE-HE film was soaked into $1 \mathrm{mM}$ phosphatidyl choline (PC) suspension (10ml) at 30 ${ }^{\circ} \mathrm{C}$ for $24 \mathrm{~h}$. The film was washed with water, and dried in vacuo (LDPE-PC-SA film). The selfassembled phospholipid layer containing stearic acid (StA) was similarly fabricated by using various ration of PC and StA suspension (LDPE-StA-PCSA film).

2.3 Immobilization of albumin onto LDPE-StAPC-SA film

The LDPE-StA-PC-SA film was soaked into $10 \mathrm{mM}$ phosphate buffer ( $\mathrm{pH} 4.0,5 \mathrm{ml}$ ) containing $48 \mathrm{mg}$ of EDC at room temperature for $2 \mathrm{~h}$. This film was washed with water.

The film was immersed into $0.1 \mathrm{mg} / \mathrm{ml}$ albumin-phosphate buffered saline (pH 7.4, $10 \mathrm{ml}$ ) at $4{ }^{\circ} \mathrm{C}$ for $48 \mathrm{~h}$. The film obtained was washed with a phosphate buffered saline ( $\mathrm{pH}$ 7.4). 


\section{Results and Discussion}

3.1 FTIR-ATR measurement of LDPE-PC-SA film

Figure 3 shows ATR-FTIR spectra of LDPE-HE and LDPE-PC-SA film. The intensity of ester and amido groups around $1700 \mathrm{~cm}^{-1}$ is similar to each other, but the peak at $1077 \mathrm{~cm}^{-1}$ ascribed to phosphate groups in the spectrum of LDPE-PC-SA film is apparently larger than that of LDPE-HE. Therefore, these results suggest that self-assembled PC layer is formed on the LDPE-PC-SA film.

3.2 Thermal stability of self-assembled phospholipid layer

We estimated the thermal stability of selfassembled phospholipid layer. After PC was adsorbed on LDPE, LDPE-VEMAC and LDPE-HE film, the ratio of PC eluted by heating from these films was measured (Fig. 4).

In the case of LDPE and LDPE-VEMAC film, PC was gradually desorbed from $30{ }^{\circ} \mathrm{C}$. On the other hand, PC adsorbed on LDPE-HE film was not eluted up to $80{ }^{\circ} \mathrm{C}$ within a detectable extent. This result suggests that the self-assembled phospholipid layer prepared by the present method is thermally stable.

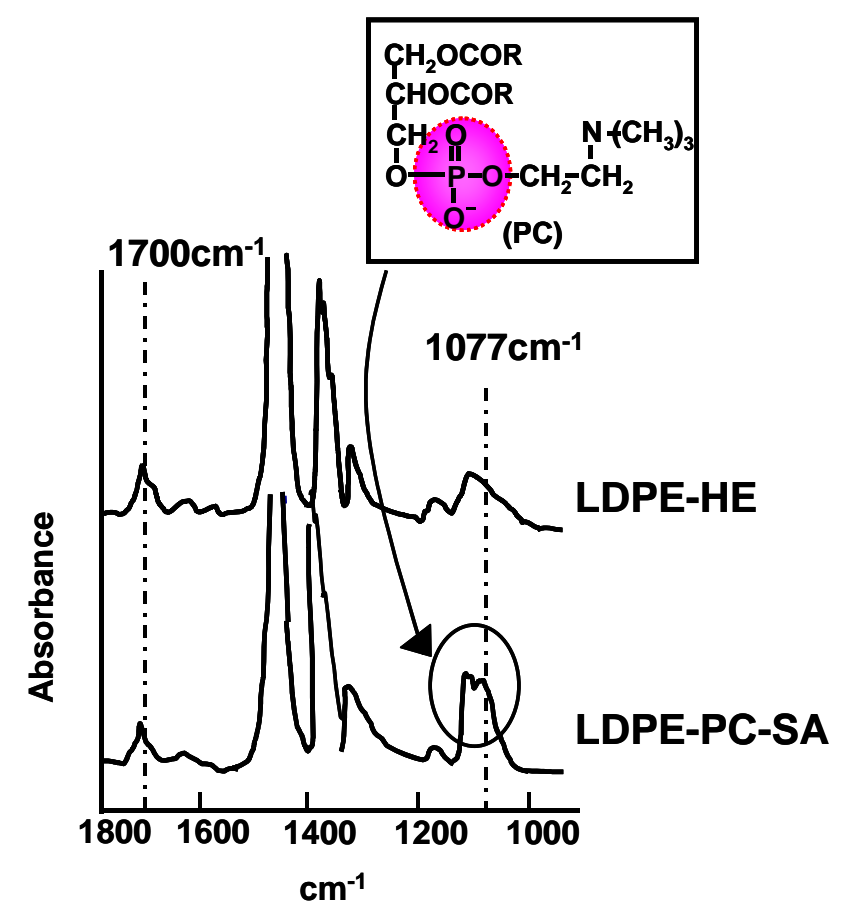

Fig.3 ATR-FTIR spectra of LDPE-HE and LDPE-PC-SA.
3.3 Immobilization of protein onto the phospholipid layer

It is difficult to bind biomolecules to PC layer directly, because PC has no efficient functional group to react with other compounds. We tried to incorporate stearic acid (StA) as a scaffold to immobilize biomolecules into the self-assembled PC layer. StA could easily be incorporated into PC layer by the immersion of LDPE-HE film into the suspension of PC and StA to obtain LDPE-StAPC-SA film.

Albumin was immobilized as a model protein onto LDPE-StA-PC-SA film with EDC (LDPE-StA-PC-SA-Albumin). The density of immobilized albumin increased with increasing that of StA in self-assembled layer, and tended to level off (Fig. 5).

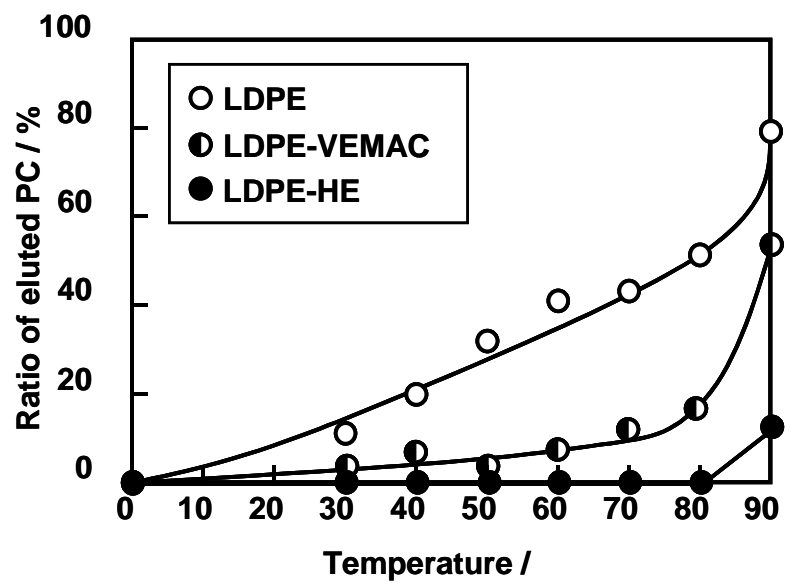

Fig.4 Plots of the elution ratio of PC adsorbed on LDPE, LDPE-VEMAC and LDPE-HE against temperature.

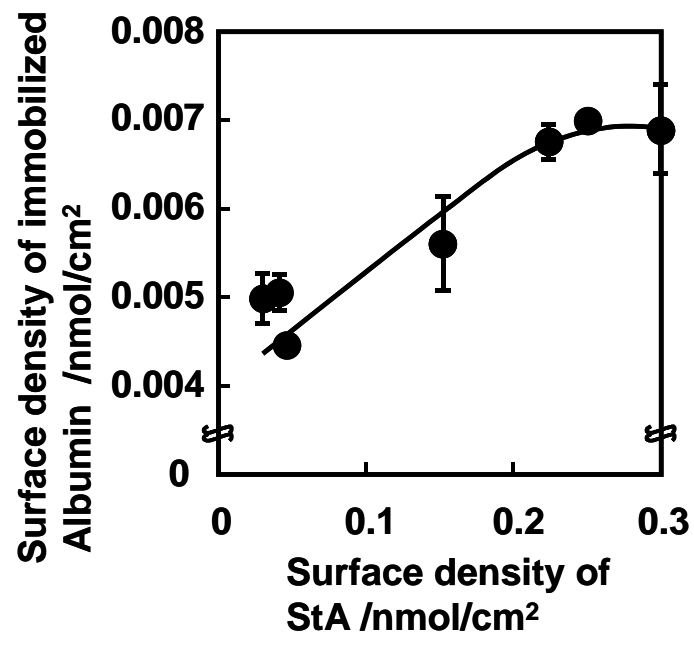

Fig. 5 Effect of surface density of StA on surface density of immobilized Albumin. 


\subsection{Fluidity of the phospholipid layer}

Half of the LDPE-StA-PC-SA-Albumin film was labeled with fluorescein-4-isothiocyanate (FITC-I). This film was immersed in water, and progressive changes in surface fluorescence were measured by confocal fluorescence microscopy.

As shown in Fig. 6, untreated area at the center and edge of the film did not fluoresce just after immersion $(0 \mathrm{~min})$. The center of untreated area fluoresced after $1 \mathrm{~h}$, but the edge did not. These results suggest that FITC-I labeled albumin moved from the treated area to the untreated. Therefore, it is reasonably assumed that this self-assembled phospholipid layer possesses fluidity.

\section{Conclusion}

The conclusions drawn from the present study can be summarized as follows.

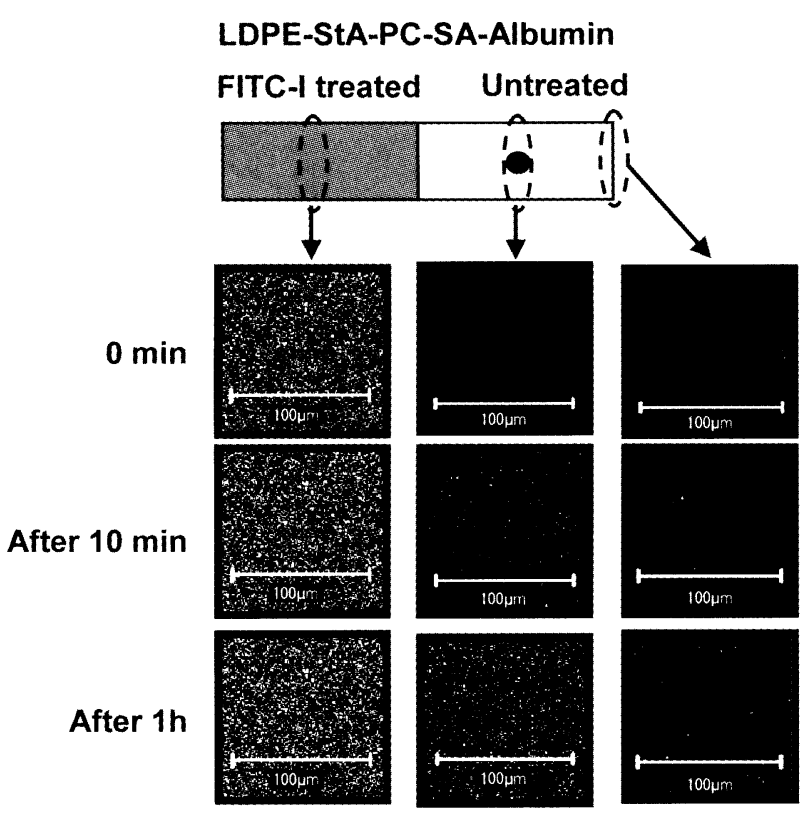

Fig. 6 Progressive changes in surface fluorescence of FITC-I labeled film. FITC-I; Fluorescein-4-isothiocyanate
The self-assembled phospholipid layer was fabricated onto LDPE-HE film by the immersion into phospholipid suspension. The self-assembled phospholipid layer was thermally stable. Fatty acid (stearic acid) could be incorporated into the self-assembled phospholipid layer, and albumin was immobilized onto this film. The amount of immobilized albumin depended on the density of stearic acid in the PC layer. It was also shown that the self- assembled phospholipid layer possessed fluidity.

It is hoped that the self-assembled phospholipid layer fabricated by the present method seems to be applicable for a novel preparation of bio-chip and bio-array immobilizing various kinds of biomolecules.

\section{References}

1. R. J. Lipshutz, S. P. A. Fodor, T. R. Gingeras, D. J. Lockhart, Nature Genet. Suppl., 21 (1991) 20.

2. M. Nouaimi, K. Möschel, H. Bisswanger, Enzyme Microb. Technol., 29 (2001) 567.

3. F. Poncin-Epaillard, G. Legeay, J. Biomate. Sci. Polymer Edn., 14 (2003) 1005.

4. M. A. Mitz, R. J. Schlueter, J. Am. Chem. Soc., 81 (1959) 4024.

5. A. Kumar, M. N. Gupta, J. Mol. Cata. B: Enzymatic 5 (1998) 289.

6. X. Chen, J. Jia, S. Dong, Electroanalysis, 15 (2003) 608.

7. M. Kuzuya, T. Yamashiro, J. Photopolym. Sci. Technol., 8 (1995) 381.

8. M. Kuzuya, Y. Matsuno, T. Yamashiro, M. Tuiki, Plasmas and Polymers, 2 (1997) 79.

9. M. Kuzuya, T. Yamashiro, S. Kondo, M. Tuiki, Plasmas and Polymers, 2 (1997) 133.

10. M. Kuzuya, T. Sawa, T. Yamashiro, S. Kondo, O. Takai, J. Photopolym. Sci. Technol., 14 (2001) 87.

11. S. Kondo, Y. Sasai, M. Kuzuya, Thin Solid Films, 515 (2007) 4136. 